\title{
NOTES ON INTEGRATION AND INTEGER SUBLATTICES
}

\author{
J. N. LYNESS, T. SØREVIK, AND P. KEAST
}

\begin{abstract}
A lattice rule is a quadrature rule over an $s$-dimensional hypercube, using $N$ abscissas located on an integration lattice. In this paper we study sublattices and superlattices of integration lattices and of integer lattices. We exploit the properties of generator matrices of a lattice to provide an easy and elegant description of the relation between a lattice and a sublattice of given order. We also obtain necessary and sufficient criteria for existence of sublattices and information about the number of these.
\end{abstract}

\section{INTRODUCTION}

This paper is concerned mainly with relations between $s$-dimensional integer sublattices and superlattices. The sort of problem considered involves finding particular lattices $\Lambda_{R}$ which contain a specified lattice $\Lambda_{B}$, i.e., $\Lambda_{B} \subseteq \Lambda_{R}$. We describe our process for doing this as factorization of $\Lambda_{B}$, since it involves factorizing an $s \times s$ generator matrix $B$ of $\Lambda_{B}$. All results about integer lattices have corresponding dual results about integration lattices, and these are collected together in $\S 5$.

These results form a part of the theory of $s$-dimensional lattice rules, a class of numerical integration rules for the hypercube. Specifically, these results may be applied to speed up the search procedure for lattice rules having particular properties, and to construct sequences of embedded lattice rules.

The $s$-dimensional lattice rule is a quadrature rule for the $s$-dimensional hypercube using a subset of order $N$ of the $N^{s}$ abscissas in $[0,1)^{s}$ required by the Cartesian product $N$-panel trapezoidal rule. These points lie on an integration lattice. The number-theoretic rules, introduced by Korobov [2], form a major subclass of lattice rules. Niederreiter [7] gives a survey and provides an extensive list of references for number-theoretic rules. Their role in numerical quadrature is examined by Haber [1]. The circumstance that properties of

Received September 21, 1989.

1980 Mathematics Subject Classification (1985 Revision). Primary 65D32.

This work was supported in part by the Applied Mathematical Sciences subprogram of the Office of Energy Research, U. S. Department of Energy, under Contract W-31-109-Eng-38.

The work of the third author was partially supported by a grant from the Natural Sciences and Engineering Research Council of Canada. This author's permanent address is Department of Mathematics, Statistics, and Computer Science, Dalhousie University, Halifax. Nova Scotia, B3H 3J5. Canada. 
number-theoretic rules are also shared by lattice rules is discussed in Sloan and Kachoyan [9].

In Lyness [3], a variant approach to the theory of $s$-dimensional lattice rules was developed based on the generator matrix $A$ of the integration lattice $\Lambda_{A}$. The points of this lattice are integer combinations of the rows of $A$. An integration lattice $\Lambda$ is one which contains the unit lattice $\Lambda_{0}$. The abscissa set of the lattice rule $Q(\Lambda)$ comprises those points of the integration lattice $\Lambda$ which lie in $[0,1)^{s}$. It was shown there that $\Lambda_{A}$ is an integration lattice if and only if $B=\left(A^{T}\right)^{-1}$ is an integer matrix.

We term the order of $B$, or of $A$ according to context,

$$
N=|\operatorname{det} B|=|\operatorname{det} A|^{-1},
$$

which is the number of abscissas in the abscissa set $A(Q) . B$ is, of course, a generator matrix of a lattice $\Lambda_{B}$, and $\Lambda_{B}$ is termed the reciprocal (polar or dual) lattice of $\Lambda_{A}$.

In this section we reintroduce briefly some of the basic theory of lattices. These results are stated and proved in many texts; for example, Newman [6, pp. 1-20], or Schrijver [8, Chapter 4]. They form a suitable background for the new results in the subsequent sections. Sections 2, 3, and 4 of the present paper are devoted to a detailed development of the integer lattice factorization theory. In $\S 5$, we give the principal dual results for integration lattices.

Definition 1.1. An $s \times s$ unimodular matrix is an integer matrix whose determinant is \pm 1 .

$U^{(i, j)}$, a unit matrix modified by having an additional unit element in the $(i, j)$ position, is clearly a unimodular matrix, as is $S^{(i)}$, a unit matrix modified by having the $(i, i)$ element replaced by -1 . It is readily verified that the $s \times s$ unimodular matrices form a group $\mathbf{U}_{s}$ under matrix multiplication, generated, for example, by the elements $U^{(i, j)}, S^{(i)}$.

Elementary unit row operations involve adding or subtracting one row to or from another, or changing the sign of a row. These may be carried out on an integer matrix by premultiplying by a unimodular matrix. It is clear that carrying out these operations on a generator matrix $B$, while naturally altering $B$, does not alter $\Lambda_{B}$. So there are many possible generator matrices $B$ of the same lattice $\Lambda_{B}$. It is convenient to define a particular form:

Definition 1.2. An $s \times s$ integer matrix $B$ is in upper triangular lattice form (utlf) if and only if

$$
\begin{array}{ll}
b_{i i} \geq 1, & i=1,2, \ldots, s, \\
b_{r c}=0, & 1 \leq c<r \leq s, \\
b_{r c} \in\left[0, b_{c c}\right), & 1 \leq r<c \leq s .
\end{array}
$$

This (or its transpose) is conventionally known as the Hermite Normal Form of $B$. The notation used here corresponds precisely to that used by Schrijver, 
except that we define our lattices in terms of rows and he does this in terms of columns.

It can be shown that any two generator matrices $B$ and $B^{\prime}$ of the same lattice $\Lambda_{B}$ are related by $B^{\prime}=U B$, where $U \in \mathbf{U}_{s}$. This forms part of the following theorem.

Theorem 1.3 (Schrijver [8, Theorems 4.1 and 4.2]). (a) Let L be a nonsingular integer matrix. There exists a unique $U \in \mathbf{U}_{s}$ such that $U L$ is in utlf.

(b) Let $B$ and $B^{\prime}$ be in utlf and be generating matrices of $\Lambda_{B}$ and $\Lambda_{B^{\prime}}$. Then $\Lambda_{B} \equiv \Lambda_{B^{\prime}}$ if and only if $B=B^{\prime}$.

This allows us to state:

Corollary 1.4. There is a 1-1 correspondence between the set of integer lattices and the set of integer matrices $B$ in utlf.

Definition 1.5. The order $N$ of a lattice $\Lambda_{B}$ is defined by $N=|\operatorname{det} B|$, where $B$ is any generator matrix.

We denote the class of $s$-dimensional lattices of order $N$ by $\mathbf{L}_{s}(N)$; the number of distinct elements in $\mathbf{L}_{s}(N)$ is denoted by $\nu_{s}(N)$; the computation of $\nu_{s}(N)$ forms the subject of [5]. Lattices of a particular order may be further classified using the diagonal elements $b_{11}, b_{22}, \ldots, b_{s s}$ of $B$, the utlf of their generator matrix.

Definition 1.6. The upper class $[\beta]=\left[b_{11}, b_{22}, \ldots, b_{s s}\right]$ of a lattice $\Lambda_{B}$ is the ordered set of the diagonal elements of the utlf of its generator matrix.

We denote the set of lattices of this upper class by $\mathbf{L}_{S}([\beta])$; the number of different lattices in a particular class $[\beta]$ is simply

$$
\nu_{s}([\beta])=b_{22} b_{33}^{2} \cdots b_{s s}^{s-1}
$$

and each of these lattices has the same order

$$
N_{B}=|\operatorname{det} B|=|[\beta]|=b_{11} b_{22} \cdots b_{s s} .
$$

We introduce class products and class factorizations in a natural manner. The relation

$$
\left[b_{11}, b_{22}, \ldots, b_{s s}\right]=\left[l_{11}, l_{22}, \ldots, l_{s s}\right]\left[r_{11}, r_{22}, \ldots, r_{s s}\right],
$$

abbreviated to $[\beta]=[\lambda][\rho]$, stands for the set of equations

$$
b_{i i}=l_{i i} r_{i i}, \quad i=1,2, \ldots, s .
$$

We refer to this relation as a class factorization of $[\beta]$, or as a class product of $[\lambda]$ and $[\rho]$.

Among some obvious consequences is

$$
|[\beta]|=|[\lambda]||[\rho]| .
$$


Finally, by extension we denote the upper class of any nonsingular integer matrix $B$ as the upper class of the lattice $\Lambda_{B}$ of which $B$ is a generating matrix.

A similar framework, using lower triangular lattice form and lower classes, exists. We have chosen the upper forms as a long discussion of lower classes would be socially unacceptable to one of the authors.

\section{Sublattices OF INTEGER LAtTices}

In this section, we derive a basic theorem about sublattices when only integer lattices are involved. We use this to investigate some of the circumstances under which a specified integer lattice $\Lambda_{R}$ has sublattices $\Lambda_{B}$ of specified order or class. In the subsequent sections, we shall treat the more complicated reverse problem, in which $\Lambda_{B}$ is specified, and we seek $\Lambda_{R}$ of specified order or class.

Theorem 2.1. Let $s \times s$ nonsingular integer matrices $B$ and $R$ be the generator matrices of $\Lambda_{B}$ and $\Lambda_{R}$, respectively. Then $\Lambda_{R} \supseteq \Lambda_{B}$ if and only if $L=B R^{-1}$ is an integer matrix.

Proof. If $\Lambda_{B} \subseteq \Lambda_{R}$, every element of $\Lambda_{B}$ is an element of $\Lambda_{R}$. Since each row $\mathbf{b}_{i}$ of $B$ is an element of $\Lambda_{R}$ we have

$$
\mathbf{b}_{i}=\lambda_{i} R, \quad i=1,2, \ldots, s,
$$

with $\lambda_{i}$ being integer. Consequently, $B=L R$, where $L$ is an integer matrix. That is, $L=B R^{-1}$. Conversely, when $B=L R$, then $\mathbf{b}_{t}=\sum \mu_{t j} \mathbf{r}_{j}$, where $\mathbf{b}_{t}$ and $\mathbf{r}_{j}$ are rows of $B$ and $R$, respectively. Then $\mathbf{b}_{t}$ is an element of $\Lambda_{R}$. Since all elements of $\Lambda_{B}$ are of the form $\sum \lambda_{t} \mathbf{b}_{t}$, it follows that all elements of $\Lambda_{B}$ are elements of $\Lambda_{R}$.

Corollary 2.2. An integer lattice $\Lambda_{R}$ of order $N_{R}$ has no sublattice $\Lambda_{B}$ of order $N_{B}$ unless $N_{B} / N_{R}$ is an integer.

Note that this theorem does not involve the utlf of the generator matrices. However, it provides only a partial answer to the question of whether the integer lattice $\Lambda_{R}$ (of order $N_{R}$ ) has a sublattice of order $N_{B}$, i.e., it does not unless $N_{R}$ is a factor of $N_{B}$.

Theorem 2.3. Let $R$ be a matrix in utlf which generates a lattice of upper class $[\rho]$. Let $L^{(i)}, i=1,2, \ldots, \nu_{s}([\lambda])$, be the distinct utlf matrices generating all lattices of upper class $[\lambda]$. Denote by $\mathbf{S}$ the set of lattices given by

$$
\left\{\Lambda\left(B^{(i)}\right) \mid B^{(i)}=L^{(i)} R, i=1,2, \ldots, \nu_{s}([\lambda])\right\} .
$$

Then:

(i) Every lattice in $\mathbf{S}$ is of upper class $[\lambda][\rho]$.

(ii) The lattices in $\mathbf{S}$ are all distinct.

(iii) If $\Lambda$ is in $\mathbf{S}$ then $\Lambda$ is a sublattice of $\Lambda(R)$.

(iv) Every lattice of upper class $[\lambda][\rho]$ which is a sublattice of $\Lambda(R)$ appears in $\mathbf{S}$ 
Proof. (i) For any $i$, since $L^{(i)}$ and $R$ are in utlf, then $B^{(i)}$ is upper triangular and has diagonal $[\lambda][\rho]$.

(ii) If $B^{(i)}, B^{(j)}$ are two upper triangular matrices generating the same lattice, then, by Theorem 1.3, $B^{(i)}=U B^{(j)}$. Thus $L^{(i)} R=U L^{(j)} R$, and so $L^{(i)}=U L^{(j)}$. However, by Theorem 1.3(a), $U=I$, which establishes that the elements of $\mathbf{S}$ are distinct.

(iii) Since $L^{(i)}=B^{(i)} R^{-1}$ is an integer matrix, then, by Theorem 2.1, $\Lambda\left(B^{(i)}\right)$ $\subseteq \Lambda(R)$. Thus, all elements of $\mathbf{S}$ are sublattices of $\Lambda(R)$.

(iv) If $\Lambda(B)$ is a sublattice of $\Lambda(R)$, then, by Theorem $2.1, L=B R^{-1}$ is an integer matrix. By Theorem 1.3 it follows that there exists a unimodular matrix $U$ such that $U L$ is in utlf. Thus, $U B=U L R$. Moreover, since $B$ is of class $[\lambda][\rho]$ and $R$ of class $[\rho]$, then $U L$ is of class $[\lambda]$. With $R$ and $L^{(i)}$ as defined in the theorem, $\Lambda(B)$ is generated by $U B$ with $B^{(j)}=L^{(j)} R$ for some $j$.

A similar theorem, in which one obtains all sublattices $\Lambda_{B_{i}}$ of $\Lambda_{R}$ that are of order $N_{L} N_{R}$, is also valid and may be proved in the same way. In this theorem, the requirement that $R$ be in utlf may be dropped.

Thus, one may construct a complete set of generator matrices of all lattices of order $N_{B}$ which are sublattices of $\Lambda_{R}$ in a straightforward way, namely,

$$
B^{(i)}=L^{(i)} R, \quad i=1,2, \ldots, \nu_{s}\left(N_{L}\right),
$$

where $N_{B}=N_{L} N_{R}$.

The next step in the theory treats

$$
B^{(i, j)}=L^{(i)} R^{(j)}, \quad \begin{aligned}
& i=1,2, \ldots, \nu_{s}\left(N_{L}\right), \\
& j=1,2, \ldots, \nu_{s}\left(N_{R}\right),
\end{aligned}
$$

where $R^{(j)}$ is a set of generating matrices in utlf of all the distinct lattices of order $N_{R}$. There are difficulties here, which are clarified in the rest of the present paper. The first is that the number of matrices $B^{(i, j)}$ constructed in this way is $\nu_{s}\left(N_{L}\right) \nu_{s}\left(N_{R}\right)$, which exceeds $\nu_{s}\left(N_{B}\right)$ unless $\left(N_{L}, N_{R}\right)=1$ (see Lyness and Sørevik [5]). Thus, in general, there must be some duplication. To continue an elementary investigation, we need the result that every lattice of order $N_{B}$ has at least one superlattice of order $N_{R}$ (when $N_{R}$ is a factor of $N_{B}$ ). This is true, and a simple direct proof based on integration lattices exists. However, in our development it is convenient to defer this to $\S 4$, where it forms part of a wider theory.

\section{SUPERLATTICES OF INTEGER LATTICES}

In this and the next section we treat the converse problem: Given a lattice $\Lambda_{B}$ (of order $N_{B}$ ), how many lattices of order $N_{R}$ (which has to be a factor of $N_{B}$ ) are superlattices of $\Lambda_{B}$ ? We treat first a more limited problem. Given a lattice $\Lambda_{B}$ of class $[\beta]$, how many lattices of class $[\rho]$ are superlattices of $\Lambda_{B}$ ? 
Clearly, the answer is zero unless a factorization $[\beta]=[\lambda][\rho]$ exists. However, even in this case the answer is not generally the same for each member of the class $[\beta]$. This is in spite of the fact that each member of class $[\rho]$ has the same number $\nu_{s}([\lambda])$ of sublattices in class $[\beta]$, as was shown in the previous section. It appears that, in general, some of these are sublattices of several different lattices of class $[\rho]$, while there are other lattices in class $[\beta]$ which have no superlattices in class $[\rho]$. This situation is described in Theorem 3.9 below.

By Theorem 2.1, the necessary and sufficient condition for $\Lambda_{R}$ to be a superlattice of $\Lambda_{B}$ is that $L=B R^{-1}$ is an integer matrix when $B$ and $R$ are any generator matrices of $\Lambda_{B}$ and $\Lambda_{R}$, respectively. So, without loss of generality, we may take both $B$ and $R$ to be in upper triangular lattice form. In this case, $L$ is also in upper triangular form (but not necessarily in utlf). Written out in full, the equations we have to satisfy in order to find $L$ and $R$ are:

$$
\begin{gathered}
b_{i i}=l_{i i} r_{i i}, \quad i=1,2, \ldots, s, \\
b_{i j}-\sum_{k=i+1}^{j-1} l_{i k} r_{k j}=l_{i i} r_{i j}+l_{i j} r_{j j}, \quad 1 \leq i<j \leq s .
\end{gathered}
$$

In our problem we have available all elements of $B$ and the diagonal elements of $R$. Two questions are: Do solutions of this set exist, and if so, how many are there?

The diagonal elements of $L$ are specified by (3.1). Now let us order the set of equations in (3.2) by columns, starting each column with the superdiagonal element and working up the column to row number 1 . Then, as long as this procedure is successful, each equation considered has only two unknowns, $r_{i j}$ and $l_{i j}$ (for some $i<j$ ), since every element on the left of (3.2) has been evaluated in an earlier calculation. Now the $i, j$ equation of (3.2) has solutions for $r_{i j}, l_{i j}$ if and only if the gcd of $l_{i i}$ and $r_{j j}$,

$$
a_{i j}=\left(l_{i i}, r_{j j}\right),
$$

divides the left-hand side of (3.2). That is,

$$
b_{i j}-\sum_{k=i+1}^{j-1} l_{i k} r_{k j} \equiv 0 \quad \bmod a_{i j} .
$$

Thus, for solutions to exist, it is necessary and sufficient that $b_{i, j}$ has the form

$$
b_{i j}=c_{i j} a_{i j}+\sum_{k=i+1}^{j-1} l_{i k} r_{k j},
$$

where $c_{i j}$ is any integer. We call a $b_{i j}$ of this form an allowable value.

There is a regularly spaced infinite set of allowable values of $b_{i j}$, each separated from its neighbors by $a_{i j}$. Thus, there are precisely $b_{j j} / a_{i j}$ such values 
in the interval $\left[0, b_{j j}\right)$. (Note that $a_{i j}$ is a factor of $r_{j j}$, which is in turn a factor of $b_{j j}$.)

However, for each allowable value of $b_{i j}$ there is an infinite set of solutions for $r_{i j}$. It is simple to show that when (3.2) has a solution $r_{i j}, l_{i j}$, it also has a solution $r_{i j}+k r_{j j} / a_{i j}, l_{i j}-k l_{i i} / a_{i j}$ for any integer $k$; moreover, all solutions for this allowable $b_{i j}$ are of this form. We are interested only in solutions $r_{i j} \in\left[0, r_{j j}\right)$. There are clearly precisely $a_{i j}$ such solutions.

We sum up the situation with respect to a single equation of set (3.2) as follows.

Lemma 3.6. When the values of all elements except $r_{i j}$ and $l_{i j}$ of the $(i, j)$ equation of (3.2) are available, there are solutions of (3.2) only for $\left(b_{j j} / a_{i j}\right)$ distinct values of $b_{i j} \in\left[0, b_{j j}\right)$, and for each of these there are $a_{i j}$ distinct solutions having $r_{i j} \in\left[0, r_{j j}\right)$.

Thus, our calculation proceeds in $s(s-1) / 2$ stages. When dealing with the $(i, j)$ equation, we encounter an inconsistency in a proportion $\left(a_{i j}-1\right) / a_{i j}$ of the values of $b_{i j}$ which could be considered, and in a proportion $1 / a_{i j}$ of the cases we find $a_{i j}$ alternative solutions. This leads immediately to the following definition and theorem.

Definition 3.7. The index $I=I([\lambda],[\rho])$ of a class factorization $[\beta]=[\lambda][\rho]$ is

$$
I=\prod_{1 \leq i \leq j \leq s} a_{i j},
$$

where, as above, $a_{i j}=\left(l_{i i}, r_{j j}\right)$.

Theorem 3.9. When a class factorization $[\beta]=[\lambda][\rho]$ exists, having index $I$, $\nu_{s}([\beta]) / I$ lattices of upper class $[\beta]$ each have I distinct superlattices of upper class $[\rho]$, while the remaining $(I-1) \nu_{s}([\beta]) / I$ lattices of upper class $[\beta]$ have no superlattices of upper class $[\rho]$.

It follows that the oniy case in which there is a superlattice of upper class $[\rho]$ for every lattice of class $[\beta]$ is a case in which $I=1$, that is, all $a_{i j}=1$. In the next section, we show that every class $[\beta]$ has a unique index-1 factorization.

We close this section with an example. We characterize the superlattices of upper class $[\rho]=[1,2,3]$ of the lattices of upper class $[\beta]=[2,6,3]$. There are $\nu_{s}([\beta])=6 \cdot 3^{2}=54$ integer lattices of this class. We find $a_{12}=2, a_{13}=1$, $a_{23}=3$, and so only $54 /(2 \cdot 3)=9$ of these have a superlattice in class $[\rho]$.

It is straightforward but tedious to find these. It transpires that their generator matrices are

$$
B=\left[\begin{array}{ccc}
2 & 2 p & q \\
& 6 & 0 \\
& & 3
\end{array}\right], \quad p=0,1,2, q=0,1,2 .
$$


Moreover, according to Theorem 3.9, each of these nine lattices have six superlattices. Thus, factorizations of the form

$$
\left[\begin{array}{ccc}
2 & 2 p & q \\
& 6 & 0 \\
& & 3
\end{array}\right]=\left[\begin{array}{ccc}
2 & l_{12} & l_{13} \\
& 3 & l_{23} \\
& & 1
\end{array}\right]\left[\begin{array}{ccc}
1 & r_{12} & r_{13} \\
& 2 & r_{23} \\
& & 3
\end{array}\right]
$$

exist, with six distinct possibilities for $R$ in utlf. The defining equations are

$$
\begin{aligned}
r_{12}+l_{12} & =p, \\
r_{23}+l_{23} & =0, \\
2 r_{13}+l_{12} r_{23}+3 l_{13} & =q .
\end{aligned}
$$

One may choose $r_{12}$ and $r_{23}$ arbitrarily. This defines $l_{12}$ and $l_{23}$; the third equation then takes the form

$$
2 r_{13}=\kappa-3 l_{13}
$$

where $\kappa$ is a known integer. There is only one integer solution of this with $r_{13} \in[0,2]$. Thus, to obtain the generator matrices of the six superlattices of $\Lambda_{B}$, when $B$ is given by (3.10), one chooses $r_{12} \in[0,1], r_{23} \in[0,2]$ and determines $r_{13}$ from (3.12).

On the other hand, with the same $[\beta]=[2,6,3]$, but with a different $[\rho]$ having the same order as the previous one, namely, $[\rho]=[2,3,1]$, we find $a_{12}=a_{13}=a_{23}=1$. Thus, every lattice $\Lambda_{B}$ of class $[\beta]$ has one and only one superlattice of class $[\rho]$. This is the special factorization discussed in the next section. (The special factorization of $B$ in (3.10) is given there.)

The wide scope of the problem is comprehended when one realizes that this example comprises a small part of the general problem of finding superlattices of order 6 of lattices of order 36 .

There are $\nu_{3}(36)=4550$ lattices $\Lambda_{B}$ of order 36 and $\nu_{3}(6)=91$ lattices $\Lambda_{R}$ of order 6 . We are taking one class of the lattices of order 36 , a class containing only 54 lattices. These may have superlattices of order 6 in any of nine different classes. Our example treats only two of these classes, the second largest, and one of the smallest.

\section{THE SPECIAL FACTORIZATION}

In the previous section we showed that, given upper class $[\beta]$ and given upper class $[\rho]$ such that a factorization $[\beta]=[\lambda][\rho]$ exists, the structure of the solution depends on the factorization index $I$, which is the product of all $a_{i j}=\left(l_{i i}, r_{j j}\right)$ for which $1 \leq i<j \leq s$. In the special case that all $a_{i j}=1$, then $I=1$ and every lattice of upper class $[\beta]$ has precisely one lattice of upper class $[\rho]$. Otherwise, this is not true. In general, of course, not all $a_{i j}=1$. However, a natural question to ask is, given $[\beta]$ and $N_{R}$, does there exist a class [ $\rho$ ] whose order is $N_{R}$ and for which all $a_{i j}=1$; if so, we shall call this factorization a special factorization.

First we note that, unless $N_{R}$ is a factor of $|[\beta]|$, there are no factorizations $[\beta]=[\lambda][\rho]$ at all. Second, we note a somewhat special case: 
Theorem 4.1. When $N_{L}=|[\beta]| / N_{R}$ and $N_{R}$ are mutually prime, there is a unique factorization $[\beta]=[\lambda][\rho]$ for which $|[\rho]|=N_{R}$, and this is a special factorization.

Proof. When $N_{L}$ and $N_{R}$ are mutually prime, $\left(l_{i i}, N_{R}\right)=1$ and $\left(r_{j j}, N_{L}\right)=$ 1. The unique solution of $b_{i i}=l_{i i} r_{i i}$ is

$$
l_{i i}=\left(N_{L}, b_{i i}\right), \quad r_{j j}=\left(N_{R}, b_{j j}\right),
$$

and $a_{i j}=1$ for all $i, j$.

In the rest of this section, we seek a special factorization in cases when $\left(N_{L}, N_{R}\right) \neq 1$.

Theorem 4.2 (The Special Factorization Recurrence). Given $[\beta]=\left(b_{11}, b_{22}\right.$, $\left.\ldots, b_{s s}\right)$ and $N$ (all positive integers), we may define

$$
\begin{gathered}
r_{11}=\left(N, b_{11}\right), \\
r_{i i}=\left(N /\left(r_{11} r_{22} \cdots r_{i-1, i-1}\right), b_{i i}\right), \quad i=2,3, \ldots, s, \\
l_{i i}=b_{i i} / r_{i i}, \quad i=1,2, \ldots,
\end{gathered}
$$

in which case

$$
[\beta]=[\lambda][\rho]
$$

and

$$
a_{i j}=\left(l_{i i}, r_{j j}\right)=1, \quad 1 \leq i<j \leq s .
$$

Proof. It is trivial, but necessary, to verify that $r_{i i}, l_{i i}$, and $a_{i j}$ as defined above are all positive integers. After this, we proceed to divide each element in (4.3) and (4.4) by $r_{i i}$ to obtain

$$
1=\left(N /\left(r_{11} r_{22} \cdots r_{i i}\right), l_{i i}\right) .
$$

Now, by definition, $a_{i j}$ is a factor of $l_{i i}$. It is also a factor of $r_{j j}$, which by (4.4) is itself a factor of $N /\left(r_{11} r_{22} \cdots r_{j-1, j-1}\right)$. Thus, when $j>i, a_{i j}$ is a factor of $N /\left(r_{11} r_{22} \cdots r_{i i}\right)$. Consequently, when $j>i, a_{i j}$ is a factor of two distinct quantities which by (4.8) are mutually prime to one another. This implies $a_{i j}=1$, establishing (4.7).

Theorem 4.9. Under the hypothesis of Theorem 4.2, $|[\rho]|=(N,|[\beta]|)$.

Proof. We define

$$
P_{i}=r_{11} r_{22} \cdots r_{i i}, \quad i=1,2, \ldots, s,
$$

and, multiplying each side of (4.4) by $P_{i-1}$, note that

$$
P_{i}=\left(N, P_{i-1} b_{i i}\right), \quad i=1,2, \ldots, s .
$$

Using this, we establish that

$$
P_{j}=\left(N, b_{11} b_{22} \cdots b_{j j}\right)
$$


by induction. The induction is properly anchored, as (4.3) establishes this with $j=1$. When (4.12) is valid with $j=i-1$, we find, using (4.11) with $i=j$, that

$$
P_{j}=\left(N,\left(N, b_{11} b_{22} \cdots b_{j-1, j-1}\right) b_{j j}\right),
$$

which reduces to (4.12) as written. Thus, (4.12) is valid for all $j \leq s$. In particular, it is valid for $j=s$, establishing the theorem.

At this stage we have shown a special factorization exists, because we can construct one using the recurrence of Theorem 4.2 with $N=N_{R}$. That theorem shows it is special. Theorem 4.9 shows that

$$
|[\rho]|=\left(N_{R},|[\beta]|\right)=N_{R} .
$$

However, we still have to show that this special factorization is unique. The following lemma is a converse of Theorem 4.2 .

Lemma 4.14. Let

$$
\begin{gathered}
b_{i i}=l_{i i} r_{i i}, \quad i=1,2, \ldots, s, \\
a_{i j}=\left(l_{i i}, r_{j j}\right)=1 \quad \forall 1 \leq i<j \leq s,
\end{gathered}
$$

and

$$
N_{R}=r_{11} r_{22} \cdots r_{s s} .
$$

Then $r_{i i}$ satisfy $(4.3),(4.4)$ above with $N=N_{R}$.

Proof. Let $h_{i}, i=1,2, \ldots, s$, be the elements generated by the recurrence, i.e.,

$$
\begin{gathered}
h_{1}=\left(N_{R}, b_{11}\right), \\
h_{i}=\left(N_{R} / r_{11} r_{22} \cdots r_{i-1, i-1}, b_{i i}\right), \quad i=2,3, \ldots, s .
\end{gathered}
$$

In view of (4.15) and (4.17), this may be rearranged as

$$
\begin{aligned}
h_{i} & =\left(r_{i i} r_{i+1, i+1} \cdots r_{s s}, r_{i i} l_{i i}\right) \\
& =r_{i i}\left(r_{i+1, i+1} \cdots r_{s s}, l_{i i}\right),
\end{aligned}
$$

and (4.16) shows that the second factor here is 1 , leaving $h_{i}=r_{i i}$; establishing the recurrence relation gives the expected values of $r_{i i}$.

We conclude from these theorems:

Theorem 4.22. Given $[\beta]$ and $N_{R}$ which is a factor of $|[\beta]|$, there exists one and only one special factorization $[\beta]=[\lambda][\rho]$ having $|[\rho]|=N_{R}$.

Proof. Theorems 4.2 and 4.9 establish that there exist special factorizations and show how to construct one of them. Lemma 4.14 shows that, if there is a special factorization, it is the one constructed.

The result mentioned at the end of $\S 2$ follows immediately. 
Theorem 4.23. When $N_{R}$ is a factor of $N_{B}$, every lattice of order $N_{B}$ has at least one superlattice of order $N_{R}$.

Proof. Since $\Lambda_{B}$ and by extension $[\beta]$ is available, we find the special class factorization $[\beta]=[\lambda][\rho]$ for which $|[\rho]|=N_{R}$, using the recurrence relation of Theorem 4.2. This ensures that $a_{i j}=1$ for all $i<j$, and so the equations $B=L R$ have a unique solution for $\Lambda_{R}$.

In numerical experiments, we have extensively used the results of this section to provide generator matrices of all lattices $\Lambda_{B}$ of order $N_{B}$ in cases when $N_{B}=N_{L} N_{R}$. Let us consider the set of matrices

$$
B^{(i, j)}=L^{(i)} R^{(j)}, \quad \begin{aligned}
& j=1,2, \ldots, \nu\left(N_{R}\right), \\
& i=1,2, \ldots, \nu\left(N_{L}\right),
\end{aligned}
$$

where $L^{(i)}$ and $R^{(j)}$ are complete sets of matrices in utlf. When $\left(N_{L}, N_{R}\right)=1$, this produces precisely the

$$
\nu_{s}\left(N_{B}\right)=\nu_{s}\left(N_{L}\right) \nu_{s}\left(N_{R}\right)
$$

matrices we need. However, when $\left(N_{L}, N_{R}\right) \neq 1$,

$$
\nu_{s}\left(N_{L}\right) \nu_{s}\left(N_{R}\right)>\nu_{s}\left(N_{B}\right),
$$

and this method, while providing all the matrices we need, provides duplicates. This duplication can be avoided if we omit all products $L^{(i)} R^{(j)}$ for which $[\lambda][\rho]$ is not a special factorization. This omission is justified by the observation that every lattice $\Lambda_{B}$ has a special factorization involving some $\Lambda_{R}$. If we include all $\Lambda_{R}$ and only special factorizations, we shall encounter every $\Lambda_{B}$ precisely once.

In $\S 3$ we characterized some of the superlattices of order $N_{R}=6$ of the superlattices of class $[\beta]=[2,6,3]$, namely those of class $\rho=[1,2,3]$. That was not a special factorization, and the somewhat complicated structure of the result is described there. Here we extend the example. With the same class $[\beta]$ and the same $N_{R}$, the special factorization obtained by Theorem 4.2 is

$$
[2,6,3]=[1,2,3][2,3,1]
$$

Since this is a special factorization, all the 54 matrices $B$ of class $[2,6,3]$ factorize into $L R$, where $L$ is of class $[1,2,3]$ and $R$ of class [2, 3, 1], and unlike the example in $\S 3$, this factorization is unique. In particular, the unique factorization of the nine matrices in $\S 3$ is

$$
\left[\begin{array}{ccc}
2 & 2 p & q \\
& 6 & 0 \\
& & 3
\end{array}\right]=\left[\begin{array}{lll}
1 & l & q \\
& 2 & 0 \\
& & 3
\end{array}\right]\left[\begin{array}{ccc}
2 & r & 0 \\
& 3 & 0 \\
& & 1
\end{array}\right],
$$

for $p=0, l=r=0$; for $p=1, l=0, r=2$; for $p=2, l=r=1$. 


\section{DuAl THEOREMS}

In this paper, we have dealt almost exclusively with integer matrices, which are the generator matrices of the reciprocal lattices of integration lattices. There is, of course, a 1-1 correspondence between elements of the set of integer lattices and elements of the set of integration lattices, which may be identified using $B=\left(A^{-1}\right)^{T}$. We shall in general identify $B=L R$ with $A=W V$, where $W=$ $\left(L^{-1}\right)^{T}$ and $V=\left(R^{-1}\right)^{T}$. It is convenient to define the order of an integration lattice as the order of the corresponding integer lattice. This corresponds to $\nu(Q)$, the order of the abscissa set $A(Q)$ of the lattice rule $Q$, which is the number of function values of $\bar{f}$ required by the rule.

Theorem 5.1. Let $V$ and $A$ be the generator matrices of integration lattices $\Lambda_{V}$ and $\Lambda_{A}$, respectively. Then $\Lambda_{V}$ is a sublattice of $\Lambda_{A}$ if and only if $V A^{-1}$ is an integer matrix.

Proof. This is a direct dual of Theorem 2.1. One finds that $V A^{-1}=L^{T}$.

Corollary 5.2. $\Lambda_{A}$ has no sublattice of order $N_{V}$, unless $\left|\Lambda_{A}\right| / N_{V}$ is an integer.

Similarly, the dual of the result encompassing (2.3) is:

Theorem 5.3. Let $\Lambda_{l}$, be an integration lattice of order $N_{R}$. Let $L^{(i)}, i=$ $1,2, \ldots, \nu_{s}\left(N_{L}\right)$ be a set of distinct integer matrices in utlf each having order $N_{L}$. Then the set

$$
A^{(i)}=\left(L^{(i)-1}\right)^{T} V, \quad i=1,2, \ldots, \nu_{s}\left(N_{L}\right),
$$

includes once only a generator matrix of every distinct integration superlattice of $\Lambda_{V}$ of order $N_{L} N_{R}$.

This result has an obvious application to lattice rule extension, i.e., the construction of lattice rules whose abscissa set contains the abscissas of a given rule. Finally, the dual of the theorem of $\S 4$ is the somewhat unexciting result:

Theorem 5.5. When $N_{V}$ is a factor of $N_{A}$, every integration lattice of order $N_{A}$ has at least one sublattice of order $N_{V}$.

\section{Conclusions}

Two related problems have been discussed in this paper. First, given an integer lattice $\Lambda(R)$ and its utlf generating matrix $R$, we have shown how to construct, without repetition, all sublattices of $R$ of specified order. The simple solution to this problem is discussed in $\S 2$ in order to provide a background for the more complicated converse problem. This is that given an integer lattice of order $N_{B}$, and given $N_{R}$ (dividing $N_{B}$ ), how many superlattices of $\Lambda(B)$ of order $N_{R}$ exist, and how can these be generated? This led to a less general problem, in which the upper class $[\beta]$ of an integer lattice $\Lambda(B)$ and a class factorization $[\lambda][\rho]=[\beta]$ where specified, and the existence of a factorization $L R=B$ was investigated. We showed that when $B$ is in utlf, and a class 
factorization $[\lambda][\rho]=[\beta]$ is specified, then the factors $L, R$ may not exist at all, or may be nonunique, or may be uniquely defined, with $R$ in utlf. The central result of this paper is the derivation of conditions on $[\lambda]$ and $[\rho]$ to ensure the existence of a unique factorization $L R=B$, with $R$ in utlf. A consequence of this result is that we can generate, in a simple manner and without repetitions, all superlattices with a given order, or a given integer lattice.

\section{BIBLIOGRAPHY}

1. S. Haber, Numerical evaluation of multiple integrals, SIAM Rev. 12 (1970), 481-526.

2. N. M. Korobov, The approximate computation of multiple integrals, Dokl. Akad. Nauk SSSR 124 (1959), 1207-1210. (Russian)

3. J. N. Lyness, An introduction to lattice rules and their generator matrices, IMA J. Numer. Anal. 9 (1989), 405-419.

4. J. N. Lyness and W. Newman, A classification of lattice rules using the reciprocal lattice generator matrix, Argonne National Laboratory Report ANL-89/20, Argonne, Illinois, 1989.

5. J. N. Lyness and T. Sørevik, The number of lattice rules, BIT 29 (1989), 527-534.

6. M. Newman, Integral matrices, Academic Press, New York, 1972.

7. H. Niederreiter, Quasi-Monte Carlo methods for multidimensional numerical integration, Numerical Integration III (G. Hämmerlin and H. Brass, eds.), ISNM, vol. 85, Birkhäuser Verlag, 1988, pp. 157-171.

8. A. Schrijver, Theory of linear and integer programming, Wiley, 1986.

9. I. H. Sloan and P. J. Kachoyan, Lattice methods for multiple integration: theory, error analysis and examples, SIAM J. Numer. Anal. 24 (1987), 116-128.

Mathematics and Computer Science Division, Argonne National laboratory, ArGONNE, ILLINOIS 60439-4801

E-mail addresses: lyness@anl-mcs.arpa

keast@cs.dal.ca

tors@eik.ii.uib.no 